\title{
Solvent dynamics: Modified Rice-Ramsperger-Kassel-Marcus theory. II. Vibrationally assisted case
}

\author{
R. A. Marcus \\ Noyes Laboratory of Chemical Physics, 127-72, California Institute of Technology, Pasadena, \\ California 91125
}

(Received 21 March 1996; accepted 27 June 1996)

\begin{abstract}
Expressions are given for a solvent dynamics-modified Rice-Ramsperger-Kassel-Marcus (RRKM) theory for clusters. The role of vibrational assistance across the transition state region is included. The usual differential equation for motion along the slow coordinate $X$ in constant temperature systems is modified so as to apply to microcanonical systems. A negative entropy term, $-S_{v}(X)$, replaces the $(1 / T) \partial U / \partial X$ or $(1 / T) \partial G / \partial X$ which appears in canonical systems. Expressions are obtained for the RRKM-type rate constant $k(X)$ and for the $S_{v}(X)$ which appear in the differential equation. An approximate solution for steady-state conditions is given for the case that the "reaction window" is narrow. The solution then takes on a simple functional form. The validity of the assumption can be checked a posteriori. Recrossings of the transition state are included and the condition under which the treatment approaches that in Part I is described.

(C) 1996 American Institute of Physics. [S0021-9606(96)51137-2]
\end{abstract}

\section{INTRODUCTION}

The field of unimolecular reactions in clusters poses new and interesting challenges. When the number of solvent molecules in a cluster is increased one expects that at a given total energy the unimolecular reaction rate will decrease, because of the increased number of degrees of freedom sharing the excess energy. Recently, some experimental results on the isomerization of trans-stilbene in hexane clusters as a function of cluster size have been obtained by Zewail and co-workers, ${ }^{1}$ who observed this decrease in rate outside the reaction threshold region. A second contributing effect to this decreased rate could be an enhanced frictional effect due to the extra number of solvent molecules in the cluster. At present, the question remains open as to which effect predominates. Both effects were included in the solvent dynamics-modified RRKM theory in Ref. 2, designated below as Part I. In the present paper, the theory is broadened to consider the case where fast (vibrational) coordinates can assist the system in crossing the transition state region. Such effects occur in some other systems, as noted below. We have discussed connections between experiment and theory in the solvent dynamics field elsewhere. ${ }^{3}$ The relation between the present formalism and that in Part I is described in a later section.

In the theory in Part I, RRKM microcanonical concepts were employed ${ }^{4}$ and Kramers, ${ }^{5}$ ideas for solvent friction effects in constant temperature systems were adapted to the microcanonical case. In such a treatment the focus is on a single coordinate, a "reaction coordinate," its friction coefficient and a possible inertial effect. For some physical situations a new feature occurs: in some chemical reactions one coordinate is "slow," i.e., only it experiences a large "friction," while the remaining coordinates are "fast." Both types of coordinates can contribute to the reaction coordinate by assisting the system in its crossing of the transition state for the reaction, and this role for the fast coordinates has been termed vibrational assistance. For constant temperature systems it has been treated for heme-ligand binding kinetics, ${ }^{6}$ electron transfer reactions, ${ }^{7-10}$ isomerization reactions, ${ }^{11,12}$ and for model and other systems. ${ }^{13-16}$ A brief review of some of this history has been given elsewhere. ${ }^{3}$ Under certain conditions (Sec. III and Ref. 13) the vibrational assistance picture reduces to a conventional overdamped case of the Kramers' type.

Vibrational assistance effects can be substantial. In electron transfer reactions they occur when there are appreciable changes in the equilibrium bond lengths or angles as a result of the reaction. In some cases the effects even cause the reaction to proceed in a time much shorter than the relaxation time of the "slow" solvent coordinate. ${ }^{7}$ Correspondingly, they cause a reaction rate to deviate from the usual Kramers' formula for the reaction rate constant. This behavior has been observed experimentally for some electron transfer reactions, 8,9 and apparently for some isomerizations. ${ }^{11} \mathrm{~A}$ third observed feature in some instances has been a decay more complicated than single exponential. For electron transfers there have been various extensions of the original treatment ${ }^{7}$ and many applications to experimental data, e.g., Refs. 8-10. They are summarized in Ref. 3.

In "vibrationally assisted reactions" the reaction can avoid the stablest region of the conventional transition state, and the literature contains many references to avoidance of that region, a phenomenon now sometimes referred to as "saddle-point avoidance," e.g., Refs. 7 and 12-14. In the present paper, we treat the problem for microcanonical systems, for use with clusters. An aim of the present paper is to provide a microcanonical reaction-diffusion differential equation for these clusters and to derive unimolecular RRKM-type expressions for the quantities appearing in them. The differential equation itself can be solved by various methods, numerical methods, for example. An analytical decoupling approximation for the solution was given for the 
corresponding constant temperature equation, valid when there is a sufficient separation of the reaction and solvent relaxation times scales. ${ }^{7,10}$ A different analytical solution, valid for a steady-state "narrow reaction window" condition, is obtained in the present paper. It is shown how the theory reduces to conventional RRKM theory when the diffusion along the slow coordinate becomes sufficiently fast, and how it and the theory in Part I are related. A separate question commented on later and particularly in Ref. 3 is that of energy redistribution to the solvent cluster.

The organization of the paper is as follows:

In Sec. II the differential equation for the microcanonical system is formulated and expressions are derived for the local unimolecular rate constant and the local entropy (density of states) appearing in the equation. The principal results are Eq. (2.1) and expressions for quantities appearing in it, such as Eqs. (2.4) and (2.7) for a local vibrational entropy $S_{v}(X)$ and density of states $\rho(X)$ and Eqs. (2.12) and (2.13) for the local rate constant $k(X)$. The relation of the overall rate constant to the usual RRKM rate constant is also described there. One approximate solution of the differential equation for steady-state conditions is given in Sec. III for reactions that occur in a narrow reaction window $\Delta$. An expression is given for $\Delta$, and a posteriori calculation can then determine whether or not the condition for this form of solution is fulfilled. The results are extended in Sec. IV to include possible recrossings of the transition state, and Eqs. (4.1) and (4.2) for the differential equation and Eq. (4.13) for the rate constant are now obtained. Several types of entropic surfaces are described in Sec. V and a concluding discussion given in Sec. VI.

\section{THEORY}

\section{A. Differential equation}

We consider a slow overdamped coordinate $X$ and treat the remaining coordinates as fast. The microcanonical probability distribution function $P(X, t)$ is assumed to satisfy the following Smoluchowski-type equation, which at the moment we may regard as plausible but heuristic:

$$
\frac{\partial P}{\partial t}=D \frac{\partial}{\partial X}\left(\frac{\partial P}{\partial X}-\frac{P}{k_{B}} \frac{\partial S_{v}(X)}{\partial X}\right)-k(X) P(X) .
$$

A derivation of the corresponding canonical (constant temperature) equation from the Fokker-Planck equation has been given for systems where all coordinates are treated classically: ${ }^{13(b)}$ An integration is performed over the fast coordinates but introducing a Kramers-Langer reaction sink term arising from a bistable potential in some domain of the slow coordinate $X$. In our case, aside from being microcanonical rather than canonical, all coordinates are treated as quantum degrees of freedom rather than classical, except for $X$ and, in the vicinity of the transition state region, a reaction coordinate $Q$. Because of the many high frequencies involved intramolecular motion in typical unimolecular reactions, a quantum description of the energy states associated with those modes is essential, and is a feature of RRKM theory. In Sec. VI we sketch briefly in physical terms how a derivation of Eq. (2.1) from a more microscopic prescription having fast quantum modes and a classical $X$-mode might proceed.

As in a corresponding microcanonical equation in Part I, the above adaptation of the canonical equation permits the equilibrium microcanonical distribution function, which in the present instance is a distribution function for $X$ in the absence of reaction, $P_{\text {eq }}(X)$, to satisfy the equation identically. In Eq. (2.1) $k(X)$ is the reaction rate constant at $X$. We have replaced the usual potential gradient term $(-1 / T) \partial U /$ $\partial X$, or the sometimes used free energy gradient term $(-1 /$ $T) \partial G / \partial X$, for constant temperature systems, by a vibrational entropic gradient $\partial S_{v}(X) / \partial X$ at the given total energy $E$ in the present microcanonical system. Throughout most of this paper the symbol for the energy $E$ is suppressed for notational brevity. We obtain below expressions for the $k(X)$ and $S_{v}(X)$. In Eq. (2.1) $D$ is a diffusion constant in $X$ space, and can, by analogy with the constant temperature literature cited in Ref. 3, be replaced by a $D(t)$ when necessary. We return to $D$ later.

Equation (2.1) assumes that at the given total energy $E$ all coordinates but $X$ are fast, so that a statistical approximation for their distribution at the given $E$ can be made at each $X$. For comparison, we note that in RRKM theory the statistical approximation is made in relating the distribution in the transition state to that of the most probable states of the reacting molecule at the given $E$. It should be stressed that the other coordinates are not thermalized at each $X$, since the system is isolated (collision-free). Instead, a statistical internal equilibration (fast coordinates) is assumed at each $X$.

We note in passing that the corresponding Smoluchowski-type equation (with a sink term) for constant temperature conditions cannot be deduced from Eq. (2.1): the experimental conditions are very different. In the constant temperature system the reactant may diffuse a short distance along the (internal) $X$ coordinate at some total en$\operatorname{ergy} E$, then undergo a collision with the other molecules, so changing its energy to $E^{\prime}$, and so on. The time history is very different from that of an isolated (constant energy) reactant which diffuses along the internal $X$ coordinate, interacting statistically with the other internal coordinates but in a collisionless manner and at a fixed total energy $E$.

\section{B. An expression for $S_{v}(X)$}

The $S_{v}(X)$ in Eq. (2.1) is related to the equilibrium microcanonical distribution function $P_{\mathrm{eq}}(X)$ :

$$
S_{v}(X)=k_{B} \ln P_{\mathrm{eq}}(X)+\text { const. }
$$

The constant does not affect, of course, the $\partial S_{v} / \partial X$ in Eq. (2.1). We can choose the constant as $S_{v}(0)-k_{B} \ln P_{\text {eq }}(0)$, where $X=0$ denotes the equilibrium value of $X$ for the reactant. The $P_{\text {eq }}(X)$ in Eq. (2.2) is the equilibrium probability density (per unit $X$ ) for the system to be at $X$ in the absence of reaction, regardless of the value of the momentum $P_{X}$ conjugate to $X$. It equals $\rho(X) d E$, the number of quantum 
states in $(E, E+d E)$ at $X$, per unit $X$, divided by the number of quantum states of the reactant, $\rho d E$, in $(E, E+d E)$ : That is,

$$
P_{\text {eq }}(X)=\rho(X) / \rho .
$$

When integrated over $X$ this $P_{\text {eq }}(X)$ is seen to be normalized to unity, since $\int \rho(X) d X=\rho$, the integral being over the region occupied by the reactant. From Eqs. (2.2) and (2.3) and this normalization we have

$$
\rho(X) / \rho=e^{S_{v}(X) / k_{B}} / \int e^{S_{v}(X) / k_{B}} d X,
$$

where the integral is over the region occupied by the reactant.

We obtain next an expression for $\rho(X)$. If $m$ denotes the $m$ th quantum state for the reactant, with energy $E_{m}$, for all coordinates but $X$, then the number of quantum states of the reactant in $(X, X+d X)$ and in the range $(E, E+d E)$ is

$$
\rho(X) d X d E=\sum_{m} d X d P_{X}(E) / h
$$

using the semiclassical expression for the number of quantum states corresponding to the phase space volume element $d X d P_{X}$. The momentum conjugate to $X, P_{X}$, is related to $E$ by

$$
E=\frac{1}{2} P_{X}^{2}+U(X)+E_{m} .
$$

Here, $U(X)$ is the potential energy at $X$ at the local equilibrium value of all remaining coordinates. For notational brevity we use mass-weighted units. In as much as $d P_{X} / d E$ equals $1 / P_{X}$, and inasmuch as $P_{X}$ for any $d E$ can be positive or negative, giving a factor of 2 in the following, Eq. (2.5) yields

$$
\rho(X)=\sum_{m} 2 / h\left|P_{X}\right|,
$$

where $P_{X}$ is obtained from Eq. (2.6).

It is readily verified that the $\rho(X)$ in Eq. (2.7) satisfies the condition $\int \rho(X) d X=\rho$ : Upon introducing Eq. (2.7) into this integral, we note that $\int\left(2 /\left|P_{X}\right|\right) d X$ equals the period of the $X$ vibration at $X=0$, and so equals $1 / \nu, \nu$ being the vibrational frequency of the $X$ motion. If there is any anharmonicity in the motion along $X$ this $\nu$ will be a function of the energy of the $X$ oscillator $E-E_{m}$, and is denoted by $\nu_{m}$. The energy $E_{m}$ resides in the other degrees of freedom. We thus have

$$
\int \rho(X) d X=\sum_{m}\left(1 / h \nu_{m}\right)=\rho,
$$

since $1 / h \nu_{m}$ is the (semiclassical) number of quantum states of the $X$ oscillator per unit energy when its energy is $E-E_{m}$. Thereby, the sum over $m$ yields $\rho$, the density of quantum states of the reactant for all coordinates, as in Eq. (2.8). When the $E_{m}$ in Eq. (2.6) varies with $X$ for fixed $m$, the $E_{m}$ 's used in the $\rho(X)$ in Eq. (2.8) are for the $X$ region which dominates in the integral there, namely $X \cong 0$.

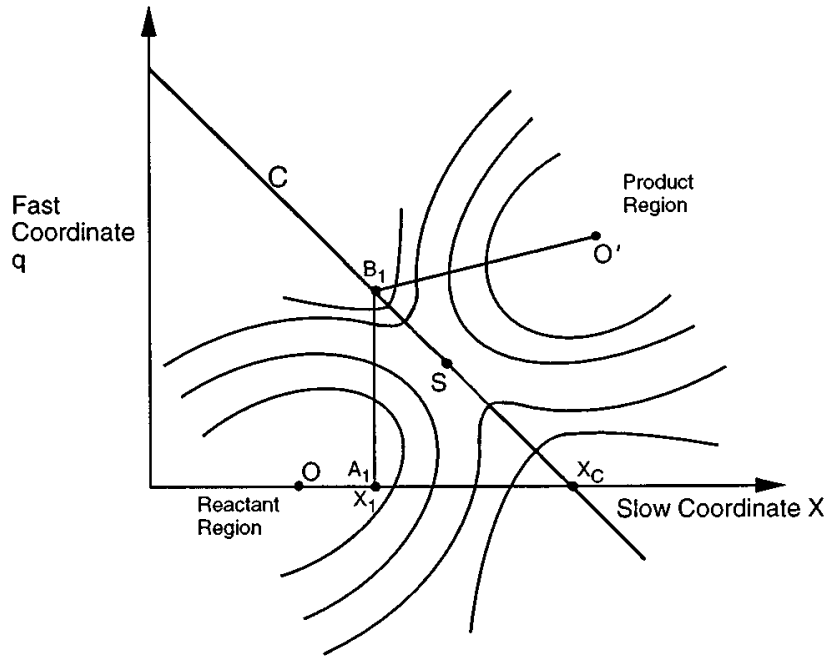

FIG. 1. Contour plots of constant (negative) vibrational entropy, $-S_{v}(X, q)$. The reaction begins at $O$ and is complete at $O^{\prime}$. The $X_{1}$ will reduce to $X_{S}$, i.e., $B_{1}$ to the saddle-point $S$, when there is no product well at an $X_{1}<X_{S}$. The wells for the negative entropy are centered at $O$ and $O^{\prime}$.

\section{An RRKM-type expression for $\boldsymbol{k}(X)$}

We obtain next an RRKM-type expression for $k(X)$. The various coordinates may assist the system in its crossing of the transition state region. To provide a purely illustrative contour plot for a system in $(X, q)$ space at the given total energy, where $q$ is some fast coordinate involved in the vibrational assistance, we introduce a vibrational entropy $S_{v}(X, q)$ in this space, related to the equilibrium microcanonical population density $P_{\text {eq }}(X, q)$ :

$$
S_{v}(X, q)=k_{B} \ln P_{\text {eq }}(X, q)+\text { const. }
$$

If one integrates over $q$ in the reactant part of space, i.e., $\int P_{\text {eq }}(X, q) d q$, one obtains the $P_{\text {eq }}(X)$ in Eq. (2.3). Thereby, $S_{v}(X, q)$ is related to $S_{v}(X)$ by

$$
\int \exp \left[S_{v}(X, q) / k_{B}\right] d q=\exp \left[S_{v}(X) / k_{B}\right] .
$$

The entropic contour plot, Fig. 1, replaces the free energy contour plot employed for constant temperature systems, e.g., in Ref. 7. While Fig. 1 is not actually used in the following treatment, it serves as a convenient visual aid. The different forms of that Figure depict graphically the various topographies discussed in Sec. V.

We first note that the reaction coordinate, denoted by $Q$, is a constant in the transition state (e.g., on the curve $\mathrm{C}$ in Figs. 1-3 below). As usual we leave open the specific definition of the reaction coordinate, but comment on it for canonical or microcanonical systems in a footnote. ${ }^{17}$ Following the well-known procedure of defining a coordinate via a family of coordinate hypersurfaces, one such member of the family corresponding to the reaction coordinate $Q$ is the transition state. On that particular hypersurface we introduce a coordinate $x$, the projection of $X$ on the transition state hypersurface, and denote the momenta conjugate to $x$ and $Q$ by 


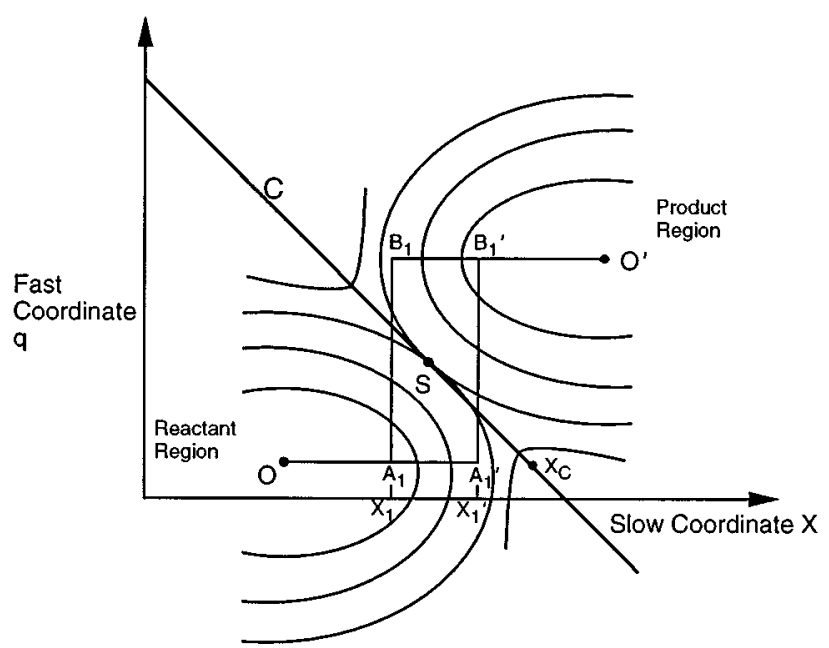

FIG. 2. Plot similar to Fig. 1, but where there is now a double well in $-S_{v}(X, q)$ at some interval of $X^{\prime}$ 's. For a symmetrical system there are two paths, $O A_{1} B_{1} O^{\prime}$ and $O A_{1}^{\prime} B_{1}^{\prime} O^{\prime}$, each contributing an equal amount to $k_{\mathrm{obs}}$, and the relative importance of each path depends upon the asymmetry of the reaction.

$p_{x}$ and $P_{Q}$. The projection is defined at the end of this section. The remaining coordinates of the transition state are treated quantum mechanically.

The semiclassical number of quantum states in the phase space volume element $d x d p_{x} d Q d P_{Q}$ is $d x d p_{x} d Q d P_{Q} / h^{2}$. To obtain the rate constant we first divide this quantity by $d Q$ to obtain the number per unit $Q$, multiply by the reaction coordinate velocity $\dot{Q}$, integrate over $P_{Q}$ and $p_{x}$, subject to the constraints given in Eq. (2.11) below, and sum over the quantum states $n$ of the transition state for all coordinates other than $x$ and $Q$. ( $Q$ is evaluated at the transition state.) We next divide by the number of quantum states, $\rho(X) d X d E$, of the reactant in that $d E$ and $d X$, and so obtain the rate constant $k(X)$ for systems crossing the transition state:

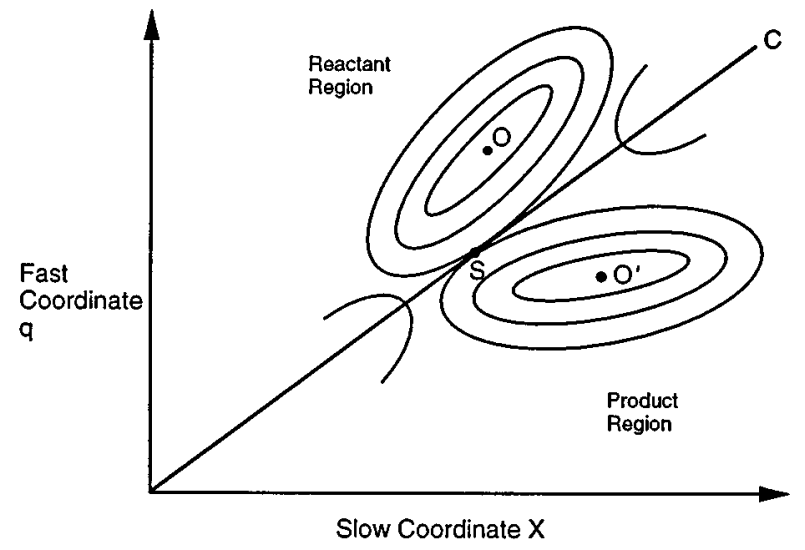

FIG. 3. Plot similar to Figs. 1 and 2. Now, however, $X_{S}$ is no longer between the $X$ for $O$ and the $X$ for $O^{\prime}$.

$$
k(X)=\left(\sum_{n} \iint \dot{Q} d P_{Q} d p_{x}\right)|d x / d X| / h^{2} \rho(X) d E .
$$

In Eq. (2.10), $\dot{Q}=P_{Q}$, using mass-weighted coordinates, and the double integral is over an area in the $\left(p_{x}, P_{Q}\right)$ space lying between $E$ and $E+d E$ and given by

$$
E \leqslant E_{n}+U_{c}(x)+\frac{1}{2} p_{x}^{2}+\frac{1}{2} P_{Q}^{2} \leqslant E+d E, \quad P_{Q} \geqslant 0,
$$

where $U_{c}(x)$ is the potential energy for the transition state (i.e., on curve $\mathrm{C}$ in Figs. 1-3) at its lowest point with respect to all coordinates but $x$; and $E_{n}$ is the energy for all coordinates but $x$ and $Q$, when the system is in the $n$th quantum state for the remaining coordinates. With the condition $P_{Q} \geqslant 0$ the integral in Eq. (2.10) is over the systems moving from the reactant's to the product's region. One finds upon integration

$$
k(X)=N(X) / h \rho(X),
$$

where

$$
\begin{aligned}
& N(X)=\sum_{n} 2 p_{x}|d x / d X| / h, \\
& p_{x}=\left\{2\left[E-E_{n}-U_{c}(x)\right]\right\}^{1 / 2},
\end{aligned}
$$

and now $p_{x}$ denotes the value of $p_{x}$ at $P_{Q}=0 . N(X)$ can be regarded as a local number (at $X$ ) of quantum states per unit $X$ for the transition state with energy equal to or less than $E$. The factor of 2 in Eq. (2.13) reflects the fact that both positive and negative $p_{x}$ contribute to $N(X)$. Equations (2.12) and (2.13) are the desired result for $k(X)$.

We conclude this section with a description of the projection $x$ of $X$ onto the transition state space and the value of $d x / d X$ in Eq. (2.13). As already noted, any coordinate (now $X)$ in a coordinate space of $N$ dimensions can be described by a family of hypersurfaces, each of dimension $N-1$. Each member of the family is associated with a different but constant value of $X$. The transition state of a reaction is a member, as previously noted, of some other family of hypersurfaces. The intersection of each member of the family of $X$ hypersurfaces with the transition state hypersurface defines a family of $N-2$ dimensional surfaces, each lying wholly within the transition state hypersurface and each having a different value of $x$. In this way, the coordinate $x$ in the transition state has been defined, apart from a scaling factor which can be chosen arbitrarily, and so the $d x / d X$ in Eq. (2.13) can be obtained.

\section{Relation of Eqs. (2.12) and (2.13) to $\boldsymbol{k}_{\text {RRKM }}$}

As a check on Eqs. (2.12) and (2.13) and also for later use in Sec. III, it is useful to consider how the usual RRKM expression for the rate constant $k_{\mathrm{RRKM}}(E)$, namely $N(E) / h \rho$, follows from those equations. When $P(X)$ has its equilibrium value, $P_{\text {eq }}(X)$, as it does in RRKM theory, we have for the equilibrium (i.e., large $D$ ) result for the observed rate constant 


$$
\begin{aligned}
k_{\mathrm{obs}}^{\mathrm{eq}} & =\int k(X) P_{\mathrm{eq}}(X) d X \\
& =\int[N(X) / h \rho] d X=\sum_{n} 2 \int p_{x} d x / h^{2} \rho,
\end{aligned}
$$

using Eq. (2.3) for $P_{\mathrm{eq}}(X)$ and Eqs. (2.12) and (2.13) for $k(X)$. The limits on this integral are now at the endpoints $p_{x}=0$, since the system is now centered near the local minimum, $x=x_{s}$, of $U_{c}(x)$. The $x$ motion has become a simple vibration. Thus, the integral in Eq. (2.14) becomes $\Sigma_{n} \oint p_{x} d x / h^{2} \rho$, where the integral is over one cycle of the $x$-vibrational motion, and it corresponds to an $x$-vibrational energy equal to $E-E_{n}-U_{c}\left(x_{s}\right)$.

The cyclic integral $\oint p_{x} d x / h$ is the classical action in units of $h$ and can be written as $\left(l+\frac{1}{2}\right)$. Semiclassically, $l$ would be an integer, the largest integer consistent with the $x$ energy being equal to or less than $E-E_{n}-U_{c}\left(x_{s}\right)$. (The balance of the energy is in the kinetic energy of the reaction coordinate $P_{Q}^{2} / 2$.) However, because of the classical derivation, this $l$ is merely a real number. Its value will be denoted by $l_{\text {max }}(n)$, since it is a function of $n$. The sum over $n$ now becomes $\Sigma_{n}\left[l_{\max }(n)+\frac{1}{2}\right]$. If $l_{\max }$ were an integer, this sum would be a step function of $E$, increasing by unity when each new $x$-quantum state becomes energetically accessible. Interestingly enough, with the presence of the $1 / 2$, one obtains instead a function of $E$ which, while continuous at each integral increment of $l$ instead of being stepped, passes through the midpoint of each vertical step in the above step function. The sum is equal to $N(E)$, the number of quantum states of the transition state, for all coordinates but the reaction coordinate, with energy equal to or less than $E$.

We thereby have

$$
k_{\mathrm{obs}}^{\mathrm{eq}}=\sum_{n}\left[l_{\max }(n)+\frac{1}{2}\right] / h \rho=N(E) / h \rho=k_{\mathrm{RRKM}}(E) .
$$

\section{E. Expression for $D$}

In Eq. (2.1) the $D$ has been inferred from various sources in the case of reactions in solution. It has been estimated, for example, from the Stokes-Einstein relation, either using the bulk viscosity or using a microviscosity, inferred from a rotational or translational diffusion constant (e.g., examples in Ref. 18). Or it has been obtained from the timedependent fluorescence dynamics Stokes shift, in the case of electron transfer systems. ${ }^{19}$ In this case $D$ may be a $D(t)$, when the relaxation is more complicated than a single exponential. The latter is sometimes replaced, instead, by a constant which is related to a relaxation time $\tau_{S}$ for the slow coordinate, or related approximately to an autocorrelation function $\int_{0}^{\infty}\langle X(0) X(t)\rangle d t /\left\langle X^{2}(0)\right\rangle$ (e.g., Refs. 10 and 20). In other papers the differential equation containing a $D(t)$ is integrated numerically. ${ }^{8,9}$ In the case of clusters, the measurement of some relaxation property in the cluster, such as a time-dependent fluorescence dynamics Stokes shift or using other ultrafast laser spectroscopy, might be used to obtain $D$ or $D(t)$. Approximate (ballpark) molecular expressions for a solute in a cluster were given in Part I for the friction coef- ficient $\zeta$ and so for $D=k_{B} T / \zeta$ in mass-weighted units. Or, molecular dynamics for the cluster could be used to estimate the autocorrelation function under microcanonical conditions.

\section{SOLUTIONS OF THE DIFFERENTIAL EQUATION}

In the case of reactions in solution, various methods have been used to solve the constant temperature analog of Eq. (2.1). For example, Agmon and Hopfield ${ }^{6}$ gave a numerical solution for a heme-ligand dissociation. Another method of numerical solution, one for electron transfers was given by Nadler and Marcus ${ }^{7}$ and one for isomerization was given by Rabinovitch and Agmon. ${ }^{12}$ For intramolecular charge transfer, Barbara ${ }^{8}$ and Yoshihara ${ }^{9}$ have integrated the differential equation numerically, sometimes with a $D(t)$ as already noted. Sumi and Marcus, ${ }^{7}$ in an analytical treatment, introduced a decoupling approximation for electron transfers, when there was an appreciable difference in time scales of the reaction and solvent relaxation. Berezhkovskii and co-workers ${ }^{13}$ have used for the steady-state case a different approximation mentioned later in Sec. IV.

Any of these methods can be used to solve the differential Eq. (2.1) for the present (microcanonical) unimolecular reaction, in conjunction with equations such as Eq. (2.4) for $S_{v}(X)$, Eq. (2.7) for $\rho(X)$, Eq. (2.12) for $k(X)$, and Eq. (2.13) for $N(X)$. In the present paper we introduce below an approximation which converts the problem to a form common in the reaction-diffusion literature: diffusion under a force to a reaction sink followed by reaction at that sink. Usually, however, the sink is at a fixed boundary in the diffusion space. In the present case, the position of the sink is determined by a maximization.

We consider the steady-state case, with a source term at $X=0$. We first note that $k(X)$ increases as $X$ increases from 0 to $X_{c}$ in Fig. 1, while $P(X)$ decreases. In this case $k(X)$ $P(X)$ has a maximum as a function of $X$, and we consider the case where this term is large near some $X, X_{1}$, but only within a narrow window, $X_{1} \pm \frac{1}{2} \Delta$. The value of $X_{1}$ will be determined by a maximization of the rate constant and the width $\Delta$ will be determined from the width of the distribution of $k(X) P(X)$ in an iterative manner described later.

Thus, we write

$$
k(X) P(X) \cong k\left(X_{1}\right) P\left(X_{1}\right) \Delta \delta\left(X-X_{1}\right),
$$

which contains $\Delta$ and a Dirac delta function. For the approximation to be applicable, it is necessary that the reaction window $\Delta$ be much smaller than the $X$ interval occupied by the reactant. A definition for $\Delta$ and a starting value in an iterative calculation for $\Delta$ is given later.

We next solve Eq. (2.1), using Eq. (3.1) and assuming a steady-state description, described above, for $P(X)$. The flux is $-D\left(\partial P / \partial X-P / k_{B} \partial S_{v} / \partial X\right)$ and it now vanishes for $X>X_{1}$. (There is no sink for $X>X_{1}$ if the reaction is along the cited path.) Integrating Eq. (2.1) from an $X<X_{1}$, after setting $\partial P / d t=0$, to an $X>X_{1}$ we have 


$$
\begin{aligned}
-D\left(\frac{\partial P}{\partial X}-\frac{P}{k_{B}} \frac{\partial S_{v}}{\partial X}\right)= & -D \exp \left(S_{v} / k_{B}\right) \\
& \times \frac{\partial}{\partial X}\left[P \exp \left(-S_{v} / k_{B}\right)\right] \\
= & k\left(X_{1}\right) P\left(X_{1}\right) \Delta \quad\left(X<X_{1}\right) .
\end{aligned}
$$

On integrating Eq. (3.2) from $X=0$ to an $X<X_{1}$, we have

$\frac{P(0)}{P(X)}=\left[\frac{k\left(X_{1}\right) \Delta}{D} \int_{0}^{X} e^{-S_{v}(X) / k_{B}} d X+e^{-S_{v}(X) / k_{B}}\right] e^{S_{v}(0) / k_{B}}$.

$P(0)$ can be determined straightforwardly from this equation by using the normalization $\int P(X) d X=1$. However, as an approximation, the neglect near $X=0$ of the first of the two terms in brackets in Eq. (3.3) yields $P(0) \cong P_{\text {eq }}(0)$ $=e^{S_{v}(0) / k_{B}} / \int e^{S_{v}(X) / k_{B}} d X$, where the integration is over the region of $X$ occupied by the reactant.

The rate constant $k_{\text {obs }}\left(X_{1}\right)$ is the probability flux (3.2) divided by $\int P(X) d X$, which is unity. Thus one sees from Eq. (3.2) that $k_{\mathrm{obs}}\left(X_{1}\right)$ equals $k\left(X_{1}\right) P\left(X_{1}\right) \Delta$ and so Eq. (3.3) yields, upon using equations such as Eqs. (2.3), (2.4), and (2.12),

$$
\frac{1}{k_{\mathrm{obs}}\left(X_{1}\right)}=\frac{1}{k_{\mathrm{act}}\left(X_{1}\right)}+\frac{1}{k_{\mathrm{diff}}\left(X_{1}\right)},
$$

where

$$
k_{\mathrm{act}}\left(X_{1}\right)=k_{\mathrm{obs}}^{\mathrm{eq}}\left(X_{1}\right)=k\left(X_{1}\right) \Delta \rho\left(X_{1}\right) / \rho=N\left(X_{1}\right) \Delta / h \rho
$$

and

$$
k_{\mathrm{diff}}\left(X_{1}\right)=D / \int_{0}^{X_{1}} \rho / \rho(X) d X .
$$

The observed rate constant $k_{\mathrm{obs}}$ for the reaction at the given $E$ is obtained from the value of $X_{1}, X_{\max }$, which maximizes the $k_{\text {obs }}\left(X_{1}\right)$ in Eq. (3.4), i.e.,

$$
k_{\mathrm{obs}}=k_{\mathrm{obs}}\left(X_{\max }\right) \text {. }
$$

The $\Delta$ in Eq. (3.5) is a reaction "window" in $X$ space. Most of the flow from reactant to product region passes through it. Since $k_{\mathrm{obs}}\left(X_{1}\right) / k_{\mathrm{obs}}\left(X_{\mathrm{max}}\right)$ is proportional to the reactive flow via the $X_{1}$ region, a reasonable value for $\Delta$, which has units of $X$, is

$$
\Delta=\int k_{\mathrm{obs}}\left(X_{1}\right) d X_{1} / k_{\mathrm{obs}}\left(X_{\max }\right),
$$

where the integral is over the region occupied by the reactant. The $\Delta$ is determined by an iterative process, where the first step in the iteration for $\Delta$ is described near the end of this section.

Equations (3.4)-(3.8) and the initial iterative equation for $\Delta$, Eq. (3.10) below, constitute the principal, results for $k_{\text {obs }}$ in this section. We note that $k_{\text {obs }}$ reduces to the $k_{\text {diff }}$ term in Eqs. (3.4) and (3.6) when $D$ becomes sufficiently small. Indeed, one could obtain that term alone by using the socalled Smoluchowski boundary condition at $X_{1}$, namely by setting $P\left(X_{1}\right)=0$ as the boundary condition at $X=X_{1}$ and then integrating Eq. (2.1) in the steady-state approximation. On the other hand, the $k_{\text {act }}\left(X_{1}\right)$ in Eq. (3.4) is the rate when $D$ is so large that $P(X)$ achieves its equilibrium value everywhere in the reactant's region. In fact, the rate under those conditions should be $\int k(X) P_{\text {eq }}(X) d X$, i.e.,

$$
\begin{aligned}
k_{\mathrm{obs}}^{\mathrm{eq}}\left(X_{1}\right) & =\int k(X) \rho(X) d X / \rho=k\left(X_{1}\right) \rho\left(X_{1}\right) \Delta / \rho \\
& =N\left(X_{1}\right) \Delta / h \rho,
\end{aligned}
$$

upon introducing Eq. (3.1) for $k(X)$. The right-hand side of Eq. (3.9) is indeed seen to be the $k_{\text {act }}\left(X_{1}\right)$ appearing in Eq. (3.5).

To see how the expression (3.9) reduces to the RRKM value when $D$ is sufficiently large, we note that $X_{\max }$ now occurs at $X_{s}$, i.e., at the $X$ corresponding to $x_{s}$, which is at the lowest value of $U_{c}(x)$ in the transition state. Thereby, the coordinate $x$ now executes a vibrational cycle. Equation (3.8) is now used for $\Delta$, and Eq. (3.9) for $k_{\text {rate }}\left(X_{1}\right)$ is introduced into it. The right-hand side of Eq. (3.8) then becomes $\int N\left(X_{1}\right) d X_{1} / N\left(X_{\max }\right)$. Using Eq. (2.13) for $N(X)$ and using the argument which led to Eq. (2.4), namely that $\int N\left(X_{1}\right) d X_{1}=N(E)$ when $X_{1}$ is centered at $X_{s}$, we obtain

$$
\Delta=N(E) / N\left(X_{\max }\right) .
$$

Since now $X_{\max }$ is $X_{s}$, Eqs. (3.7), (3.9), and (3.10), with $X_{1}$ written as $X_{\text {max }}$, yield

$$
k_{\mathrm{obs}}^{\mathrm{eq}}\left(X_{\max }\right)=N(E) / h \rho,
$$

which is the RRKM value.

We comment, finally, on the general evaluation of $\Delta$, which is determined self-consistently from Eqs. (3.4), (3.5), and (3.8), noting that $\Delta$ appears explicitly in the $k_{\text {act }}\left(X_{1}\right)$. A first estimate of $\Delta$ can be made using the value in one limiting regime, namely, when $1 / k_{\text {diff }}\left(X_{1}\right)$ can be neglected in Eq. (3.4). In that case $\Delta$ is given by Eq. (3.10). This $\Delta$ can then be used in Eq. (3.5) as a first step in an iterative scheme involving Eqs. (3.4)-(3.6).

We remark in passing, that the properties of $k_{\text {act }}\left(X_{1}\right)$ in Eq. (3.5) are determined by properties of the transition state, such as $N\left(X_{1}\right)$, while the properties of $k_{\text {diff }}\left(X_{1}\right)$ in Eq. (3.6) are determined only by the properties of the reactant, such as $D$ and $\rho(X)$.

\section{INCLUSION OF RECROSSINGS}

We consider now the case where recrossings of the transition state occur. Instead of Eq. (2.1) we now have two equations, one for the probability density of the reactant $A$ and one for that of the product $B$. The "back reaction" term, $k_{b}(X) P_{b}(X)$, provides the recrossings:

$$
\begin{aligned}
\frac{\partial P_{a}}{\partial t}= & D_{a} \frac{\partial}{\partial X}\left(\frac{\partial P_{a}}{\partial X}-\frac{P_{a}}{k_{B}} \frac{\partial S_{v}^{a}}{\partial X}\right)-k_{a}(X) P_{a}(X) \\
& +k_{b}(X) P_{b}(X),
\end{aligned}
$$




$$
\begin{aligned}
\frac{\partial P_{b}}{\partial t}= & D_{b} \frac{\partial}{\partial X}\left(\frac{\partial P_{b}}{\partial X}-\frac{P_{b}}{k_{B}} \frac{\partial S_{v}^{b}}{\partial X}\right)-k_{b}(X) P_{b}(X) \\
& +k_{a}(X) P_{a}(X) .
\end{aligned}
$$

The values of $k_{a}(X)$ and $k_{b}(X)$ are given by Eq. (2.12), with appropriate subscripts:

$$
k_{a}(X)=N(X) / h \rho_{a}(X), \quad k_{b}(X)=N(X) / h \rho_{b}(X),
$$

and $N(X)$ is given by Eq. (2.13), as before. Equations (2.12) and (4.3) presume a local entropic $\left[-S_{v}^{b}(X, q)\right]$ well for the product upon crossing the transition state hypersurface at the given $X$. The $S_{v}^{a}(X) / k_{B}$ and $S_{v}^{b}(X) / k_{B}$ can, using Eqs. (2.2) and (2.3), be written as

$$
\begin{aligned}
& S_{v}^{a}(X) / k_{B}=\ln \rho_{a}(X)+C, \\
& S_{v}^{b}(X) / k_{B}=\ln \rho_{b}(X)+C,
\end{aligned}
$$

where the constant $C$ is chosen to be the same, so that the two $S_{v}$ 's are calculated relative to the same zero. We take $C=S_{v}^{a}(0)-k_{B} \ln \rho_{a}(0)$, but the constant does not enter into Eqs. (4.1) and (4.2). As before, the value of $\int \rho_{a}(X) d X$, integrated over the reactant's region, equals $\rho_{a}$, the density of states of the reactant. We have, thereby,

$$
e^{S_{v}^{a}(X) / k_{B}} / \int e^{S_{v}^{a}(X) / k_{B}} d X=\rho_{a}(X) / \rho_{a} .
$$

From Eqs. (4.4)-(4.6a), we have

$$
e^{S_{v}^{b}(X) / k_{B}} / \int e^{S_{v}^{a}(X) / k_{B}} d X=\rho_{b}(X) / \rho_{a} .
$$

Equations (4.1) and (4.2) can again be solved by a variety of methods, and we explore next the narrow reaction window method introduced in Sec. III. We again consider the steady-state case, $\partial P_{a} / \partial t=\partial P_{b} / \partial t=0$, and use Eq. (3.1) for each $k(X)$. The fluxes $J(X)$ are given by

$$
J^{a}(X)=-D_{a}\left(\frac{\partial P_{a}}{\partial X}-\frac{P_{a}}{k_{B}} \frac{\partial S_{v}^{a}}{\partial X}\right)
$$

and

$$
J^{b}(X)=-D_{b}\left(\frac{\partial P_{b}}{\partial X}-\frac{P_{b}}{k_{B}} \frac{\partial S_{v}^{b}}{\partial X}\right) .
$$

The steady-state condition for the net forward reaction can again be imagined as arising from a continuous source of $A$ at $X=0$ and a sink of equal strength for $B$ at $X=0^{\prime}$ (Figs. 1 or 2). The steady-state rate constant is then the probability flux of $A$, divided by $\int P_{a}(X) d(X)$, which is unity. The rate constant is given later in Eq. (4.12).

With a delta function form of rate constants in Eq. (3.1), integration of Eqs. (4.1) and (4.2) over the appropriate $X$ intervals shows that $J^{a}(X)$ and $J^{b}(X)$ are piecewise constant: $J^{a}(X)=J^{a}(0) \quad$ for $\quad 0 \leqslant X<X_{1}, \quad J^{b}(X)=J^{b}\left(0^{\prime}\right)$ for $0^{\prime} \geqslant X>X_{1}$, and since there is no sink or source for $A$ for $X>X_{1}$ and none for $B$ for $X<X_{1}$ (cf. Figs. 1 or 2), $J^{a}(X)=0$ for $X>X_{1}$ and $J^{b}(X)=0$ for $X<X_{1}$.
Integrating Eqs. (4.1) and (4.2) over a region containing $X=X_{1}$, using Eq. (3.1), steady-state conditions, $\partial P_{a} / \partial t=\partial P_{b} / \partial t=0$, and the above results for the $J$ 's, we have

$$
J^{a}(0)=\left[k_{a}\left(X_{1}\right) P_{a}\left(X_{1}\right)-k_{b}\left(X_{1}\right) P_{b}\left(X_{1}\right)\right] \Delta=J^{b}\left(0^{\prime}\right),
$$

where $k_{a}\left(X_{1}\right)$ or $k_{b}\left(X_{1}\right)$ is the $k\left(X_{1}\right)$ in Eq. (3.1) with an $a$ or $b$ subscript.

We introduce the symbol $J$ to denote this common value:

$$
J=J^{a}(0)=J^{b}\left(0^{\prime}\right) .
$$

Integrating of Eq. (4.7) from $X=0$ to an $X<X_{1}$, noting that $J^{a}(X)=J$ in that region, yields

$$
\begin{aligned}
& P_{a}(0) e^{\left[S_{v}^{a}(X)-S_{v}^{a}(0)\right] / k_{B}-P_{a}(X)} \\
& =\frac{J}{D_{a}} e^{S_{v}^{a}(X)} \int_{0}^{X} e^{-S_{v}^{a}(X) / k_{B}} d X .
\end{aligned}
$$

The $P_{a}(0)$ can be determined from this equation, using a normalization $\int P_{a}(X) d X=1$, by integrating Eqs. (4.10) over the region occupied by $A$. However, using the equilibrium approximation in the vicinity of $X=0$, i.e., neglecting the right hand side in Eq. (4.10) when $X \cong 0, P_{a}(0)$ equals $\exp \left(S_{v}^{a}(0) / k_{B}\right) / \int \exp \left[S_{v}^{a}(X) / k_{B}\right] d X$.

Integrating Eqs. (4.8) from $X=X_{1}$ to $X=0^{\prime}$, noting that $J^{b}(X)=J$ in this interval, and setting $P_{b}\left(0^{\prime}\right)=0$, e.g., by using a sufficiently strong sink to avoid the back-reaction $B \rightarrow A$ in this calculation of a forward rate constant, yields

$$
P_{b}\left(X_{1}\right)=\frac{J}{D_{b}} \int_{X_{1}}^{0^{\prime}} e^{\left[S_{v}^{b}\left(X_{1}\right)-S_{v}^{b}(X)\right] / k_{B}} d X .
$$

The rate constant for a given $X_{1}, k_{\mathrm{obs}}\left(X_{1}\right)$, is defined by

$$
k_{\mathrm{obs}}\left(X_{1}\right)=J \text {, }
$$

divided by $\int P_{a}(X) d X$, which is unity.

Equation (4.10), valid for $X<X_{1}$ is, by continuity, valid at $X=X_{1}$. Equations (4.9)-(4.12) then yield, upon using Eq. (4.10) at $X=X_{1}$,

$$
\frac{1}{k_{\mathrm{obs}}\left(X_{1}\right)}=\frac{1}{k_{\mathrm{act}}\left(X_{1}\right)}+\frac{1}{k_{\mathrm{diff}}^{a}\left(X_{1}\right)}+\frac{1}{k_{-\mathrm{diff}}^{b}\left(X_{1}\right) K\left(X_{1}\right)},
$$

where $k_{\text {act }}\left(X_{1}\right)$ is defined by Eq. (3.5), $k_{\text {diff }}^{a}\left(X_{1}\right)$ by Eq. (3.6), with $D, \rho$ and $\rho(X)$ written as $D_{a}, \rho_{a}$, and $\rho_{a}(X)$, and $k_{- \text {diff }}^{b}\left(X_{1}\right) K\left(X_{1}\right)$ is defined by

$$
\begin{aligned}
& k_{-\mathrm{diff}}^{b}\left(X_{1}\right)=D_{b} / \int_{X_{1}}^{0^{\prime}}\left[\rho_{b}\left(X_{1}\right) / \rho_{b}(X)\right] d X, \\
& K\left(X_{1}\right)=\left[k_{a}\left(X_{1}\right) / k_{b}\left(X_{1}\right)\right] \rho_{a}\left(X_{1}\right) / \rho_{a} .
\end{aligned}
$$

The rate constant $k_{\mathrm{obs}}$ is again given in terms of $k_{\mathrm{obs}}\left(X_{1}\right)$ by Eq. (3.7), but using Eq. (4.13) instead of Eq. (3.4).

One can see that Eqs. (4.14) and (4.15) contain an equilibrium constant $K\left(X_{1}\right)$ for formation of $B\left(X_{1}\right)$ from $A$, multiplied by a diffusion rate $k_{- \text {diff }}^{b}\left(X_{1}\right)$ for forming $B$ from $B\left(X_{1}\right)$. When $k_{b}\left(X_{1}\right) / k_{a}\left(X_{1}\right)$ is sufficiently large, the last 
term in Eq. (4.13) becomes very large, and so it dominates the other two terms in Eq. (4.13), and then $k_{\text {obs }}\left(X_{1}\right)$ becomes very small. One can see the physical origin of this smallness: When the equilibrium constant between $A$ and $B\left(X_{1}\right)$ favors very much the side of $A$, the recrossings are especially extensive and the $k_{\mathrm{obs}}\left(X_{1}\right)$ is small.

The origin of the structure of Eq. (4.13) is seen clearly from the scheme $A \rightleftarrows A\left(X_{1}\right) \rightleftarrows B\left(X_{1}\right) \rightarrow B$, upon using a steady-state approximation for $A\left(X_{1}\right)$ and $B\left(X_{1}\right)$, the concentrations of $A$ and $B$, per unit $X$, at $X_{1}$. If the forward and reverse rate constants of the first step are denoted by $k_{1}$ and $k_{-1}$, of the second step by $k_{2}$ and $k_{-2}$, and if the forward rate constant of the final step is $k_{3}$ one obtains

$$
\frac{1}{k_{\mathrm{obs}}}=\frac{1}{k_{2} K_{1}}+\frac{1}{k_{1}}+\frac{1}{k_{3} K_{1} K_{2}},
$$

where $K_{i}\left(=k_{i} / k_{-i}\right)$ is the equilibrium constant for $i=1,2$. The structure of Eq. (4.16) is identical to that of Eq. (4.13). We note that in this model leading to Eq. (4.16) there are net flows in the $A$ region and in the $B$ region, and that both flows are equal. The same behavior occurs in Eq. (4.9b), which leads to Eq. (4.13).

\section{ENTROPIC SURFACES}

There are several different types of arrangement of the entropic wells. We have plotted $-S_{v}(X, q) / k_{B}$ instead of $S_{v}(X, q) / k_{B}$, so that the wells in the surface are regions of stability, and vice versa. One type of surface is depicted in Fig. 1. Here, there is no double well at any $X_{1}$, apart from the $X$ at the saddle-point, $X_{S}$. If one introduced the idea of an intermediate $B_{1}$ for this case, it would now be so short-lived, because of recrossings, that $X_{1}$, the $X$ which maximizes $k_{\text {obs }}(X)$, is then expected to become $X_{S}$. One then would use a one-coordinate treatment, such as that in Part I.

A second type of $-S_{v}(X, q)$ surface has two wells at a suitable range of $X_{1}$ 's, as in Fig. 2. We consider this system next. When the reaction is symmetric, the rate constant for the path $0 \rightarrow A_{1} \rightarrow B_{1} \rightarrow 0^{\prime}$ equals that for the path $0^{\prime} \rightarrow B_{1} \rightarrow A_{1} \rightarrow 0$. By symmetry, there must be a second forward path $0 \rightarrow A_{1}^{\prime} \rightarrow B_{1}^{\prime} \rightarrow 0^{\prime}$, whose properties and rate constant are identical to those of the path $0^{\prime} \rightarrow B_{1} \rightarrow A_{1} \rightarrow 0$. In comparing these two paths from 0 to $0^{\prime}$, we note that though equal in rate constant, the first path might, at first glance seem to be the more probable one, since the first step, 0 to $A_{1}$, compared with 0 to $A_{1}^{\prime}$, involves less uphill diffusion along $X$. On the other hand, the next step is more uphill from $A_{1}$ to $B_{1}$ than from $A_{1}^{\prime}$ to $B_{1}^{\prime}$. For that reason, the recrossing from $B_{1}$ to $A_{1}$ is greater than that for $B_{1}^{\prime}$ to $A_{1}^{\prime}$. A net result, one can see by microscopic reversibility, is that the two paths contribute equally to the rate constant, and the total forward rate constant is the sum of the two. The rate constant for the second path is given by an expression similar to Eq. (4.13), but now the $X_{1}$ occurs at $A_{1}^{\prime}$ instead of at $A_{1}$, and we denote this second value of $X_{1}$ by $X_{1}^{\prime}$. When the reaction is made asymmetric, by making it increasingly downhill, the $X_{1}$ path becomes increasingly dominant over the $X_{1}^{\prime}$ path and vice versa if the reaction is made increasingly uphill.
We now have a $k_{\text {obs }}$ at the given $E$ that is given by

$$
\begin{aligned}
k_{\mathrm{obs}}= & \max \left[k_{\mathrm{obs}}\left(X_{1}\right)+k_{\mathrm{obs}}\left(X_{1}^{\prime}\right)\right] \\
& X_{1}, X_{1}^{\prime}
\end{aligned}
$$

for both the symmetric and the asymmetric case.

A third type of surface topography is depicted in Fig. 3. Here, the system diffuses along the $X$ coordinate towards the origin and then, after the reaction occurs, diffuses in the reverse direction along the product's well. In the case of an intramolecular transfer of a light atom between two parts of a molecule, $\alpha$ and $\beta$, for example, one expects that if $X$ were the $\alpha \beta$ separation distance, the contours in a mass-weighted coordinate system would resemble those in Fig. 3 in the twodimensional subspace being considered, where the angle between the $X$ axis and line $C$ would be quite small.

\section{DISCUSSION}

The main results of the present paper, given in Sec. II, are the microcanonical reaction-diffusion equations, Eqs. (2.1), (4.1), and (4.2), and the expressions for $k(X), \rho(X)$, and $S_{v}(X)$ which appear in Secs. II and IV. With these expressions, the differential equations can be integrated numerically, with a replacement of the $D$ by a $D(t)$ when needed, as in the canonical case, or alternatively, using some analytic approximation. One example of such an approximation is described in Sec. IV, for the case that the reaction occurs only in a small interval $\left(X_{1} \pm \frac{1}{2} \Delta\right)$. As discussed there, recrossings of the transition state can play a major role in determining the value of $X_{1}$, and also there can be two such paths. Further, the surface topography is important in determining whether or not there is a saddle-point avoidance, i.e., whether or not $X_{1}=X_{S}$, and thereby in determining whether the simple treatment in Part I can be used for microcanonical systems instead of the present one.

A detailed and insightful discussion of surface topography and localized reaction paths has been given by Berezhkovskii and Zitserman ${ }^{13}$ for reactions at a given temperature. They consider at each $X$ an escape time to the product well and a solvent relaxation time. The $X$ where the two are equal is where the reaction is presumed to occur. They note that if there is no bistable potential before reaching $X_{S}$, the value of $X$ at the saddle-point, the treatment reduces to a multidimensional Kramers-Langer type result. The physical effects presented in Ref. 13 regarding escape time and solvent relaxation time are closely related to the ratio of $k_{\text {diff }}$ $\left(X_{1}\right) / k_{\text {act }}\left(X_{1}\right)$ in the present Eq. (3.4). Recrossings were also extensively discussed in Ref. 13.

We have noted elsewhere ${ }^{3}$ that the current experimental data are insufficient to distinguish between the Kramersmodified form of RRKM theory for unimolecular reactions presented in Part I and the one slow coordinate-many fast coordinate treatment in the present paper. The acquisition and the use of auxiliary information on solvent dynamics, which has been helpful in solution (e.g., for electron transfers and discussed in Ref. 3), would be helpful for clusters also. As discussed there the choice of suitable solutes could minimize the effects of solvent friction and so emphasize, 
and be instructive of, the energy-sharing role of the solvent molecules in the cluster. For the present, as noted in Part I, the energy $E$, largely arising in Ref. 1 from photoexcitation, can be regarded as distributed in one of two limiting wayssolely in the solute or throughout the cluster. Further experiments can be expected to distinguish between these possibilities and determine the time scale for proceeding from one to the other.

In concluding this section, we sketch briefly in physical terms how Eq. (2.1) could be obtained from a more molecular description, beginning with a mixed quantum-classical description of the modes. For all modes but $X$ a quantum basis set of zeroth-order states would be introduced, whose nature could vary with the value of $X$. [In that case the $E_{m}$ in Eq. (2.6) becomes $E_{m}(X)$.] For example, some bending vibrations for the stable configurations of the reactant become, in the case of a nearly loose transition state, almost free rotations. The zeroth-order states are coupled to each other and to the classically treated coordinate $X$. The equations of motion would describe the dynamics of this zeroth-order basis set and of $X$. At each value of $X$ one would average over the fast coordinates, e.g., by assuming a statistical equilibrium for those coordinates and, in the present (overdamped) case, for $P_{X}$. In that case, the change in the gradient of the density of the zeroth-order states, $\rho(X)$, provides a "thermodynamic" force for the motion along $X$. In this way, more or else by analogy with Ref. 3(a) but for a constant energy system and in quantum terms, one could obtain Eq. (2.1) without the reaction term. In addition to this local statistical equilibrium at each $X$, one then makes a statistical assumption for systems crossing the transition state region $C$ at that $X$, and assumes, but only in the vicinity of $C$, a classical description for the motion along the reaction coordinate $Q$. This reaction sink at each $X$ provides the $k(X)$ term in Eq. (2.1).

\section{ACKNOWLEDGMENTS}

It is a pleasure to acknowledge the support of the National Science Foundation and the Office of Naval Research in the support of this research, and to thank a reviewer for thoughtful comments.

\footnotetext{
${ }^{1}$ A. A. Heikal, S. H. Chong, J. S. Baskin, and A. H. Zewail, Chem. Phys. Lett. 242, 380 (1995).

${ }^{2}$ R. A. Marcus, Chem. Phys. Lett. 244, 10 (1995).

${ }^{3}$ R. A. Marcus, Adv. Chem. Phys. (in press).

${ }^{4}$ R. A. Marcus, in Chemische Elementarprozesse, edited by H. Hartmann (Springer, Berlin, 1968), p. 348; D. M. Wardlaw and R. A. Marcus, Adv. Chem. Phys. 70, 231 (1988); R. G. Gilbert and S. C. Smith, Theory of Unimolecular and Recombination Reactions (Blackwell, Oxford, 1990).

${ }^{5}$ H. A. Kramers, Physica 7, 284 (1940).

${ }^{6}$ N. Agmon and J. J. Hopfield, J. Chem. Phys. 78, 6947 (1983); 80, 592 (E) (1984); 79, 2042 (1983).

${ }^{7}$ H. Sumi and R. A. Marcus, J. Chem. Phys. 84, 4894 (1986); W. Nadler and R. A. Marcus, ibid. 86, 3096 (1987); H. Sumi and R. A. Marcus, J. Electroanal. Chem. 204, 59 (1986).

${ }^{8}$ G. C. Walker, E. Akesson, A. E. Johnson, N. E. Levinger, and P. F. Barbara, J. Phys. Chem. 96, 3728 (1992); G. C. Walker, P. F. Barbara, S. K. Doorn, Y. H. Dong, and J. T. Hupp, ibid. 95, 5712 (1991); E. Akesson,
}

G. C. Walker, and P. F. Barbara, J. Chem. Phys. 95, 4188 (1991); D. K. Phelps and M. J. Weaver, J. Phys. Chem. 96, 7187 (1992); J. D. Simon and R. Doolen, J. Am. Chem. Soc. 114, 4861 (1992); J. P. Collin, A. Harriman, V. Heitz, F. Odobel, and J. P. Sauvage, ibid. 116, 5679 (1994).

${ }^{9}$ K. Yoshihara, K. Tominaga, and Y. Nagasawa, Bull. Chem. Soc. Jpn 68, 696 (1995); Y. Nagasawa, A. P. Yartsev, K. Tominaga, P. B. Bisht, A. E. Johnson, and K. Yoshihara, J. Phys. Chem. 99, 653 (1995); Y. Nagasawa, A. P. Yartsev, K. Tominaga, A. E. Johnson, and K. Yoshihara, J. Chem. Phys. 101, 5717 (1994); K. Yoshihara, Y. Nagasawa, and A. Yartsev, J. Mol. Liq. 65, 59 (1995).

${ }^{10}$ J. C. Rasaiah and J. J. Zhu, J. Chem. Phys. 98, 1213 (1993); J. J. Zhu and J. C. Rasaih, ibid. 96, 1435 (1992); 95, 3325 (1991); M. Morillo and R. J. Cukier, ibid. 89, 6736 (1988).

${ }^{11}$ H. Sumi and T. Asano, Chem. Phys. Lett. 240, 125 1995; J. Chem. Phys. 102, 9565 (1995); H. Sumi, J. Phys. Chem. 95, 3334 (1991); T. Asano, H. Furuta, and H. Sumi, J. Am. Chem. Soc. 116, 5545 (1994).

${ }^{12}$ N. Agmon and R. Kosloff, J. Phys. Chem. 91, 1988 (1987); S. Rabinovich and N. Agmon, Chem. Phys. Lett. 182, 336 (1991). A Smoluchowski-type equation for a different process, namely for bimolecular diffusioncontrolled reactions with a distributed reaction sink has been given by G. Wilemski and M. Fixman, J. Chem. Phys. 58, 4009 (1973).

${ }^{13}$ (a) A. M. Berezhkovskii, S. A. Dudko, and V. Yu. Zitserman, Chem. Phys. 187, 275 (1994); A. M. Berezhkovskii and V. Yu. Zitserman, Chem. Phys. Lett. 172, 235 (1990); A. M. Berezhkovskii, Chem. Phys. 164, 331 (1992); A. M. Bereshkovskii and V. Yu. Zitserman, ibid. 164, 341 (1992); J. Phys. A: Math. Gen. 25, 2077 (1992); Physica A 166, 585 (1990); A. M. Berezhkovskii and S. A. Dudko, J. Chem. Phys. 100, 5949 (1994); (b) A. M. Berezhkovskii and V. Yu. Zitserman, Physica A 187, 519 (1992).

${ }^{14}$ M. Berkowitz, J. D. Morgan, and J. A. McCammon, J. Chem. Phys. 79, 5563 (1983); S. H. Northrup and J. A. McCammon, ibid. 78, 987 (1983).

${ }^{15}$ E. Pollak, J. Chem. Phys. 85, 865 (1986); 93, 1116 (1990); 95, 533 (1991); E. Pollak, S. C. Tucker, and B. J. Berne, Phys. Rev. Lett. 65, 1399 (1990); S. Tucker and E. Pollak, J. Stat. Phys. 66, 975 (1992); E. Pollak, A. M. Berezhkovskii, and Z. Schuss, J. Chem. Phys. 100, 334 (1994); A. M. Berezhkovskii, E. Pollak, and V. Yu. Zitserman, ibid. 97, 2422 (1992).

${ }^{16}$ A. M. Frishman, A. M. Berezhkovskii, and E. Pollak, Phys. Rev. E 49, 1216 (1994); A. M. Berezhkovskii, A. M. Frishman, and E. Pollak, J. Chem. Phys. 101, 4778 (1994).

${ }^{17}$ Classically, the transition state is a hypersurface in the classical phase space and, as Wigner has pointed out [E. Wigner, Trans. Faraday Soc. 34, 29 (1938)] the best choice for this hypersurface is the one with the fewest recrossings by trajectories. A family of hypersurfaces is defined, hopefully where some member has few or negligible recrossings, and the family serves to define the reaction coordinate $Q$. In practice, only in the simplest case, namely where the potential energy is a quadratic function of the coordinates in the transition state region, can $Q$ readily be determined, namely as the coordinate associated with the mode of imaginary frequency at the saddle point. However, for more general systems, finding the best $Q$ for reactions involving rupture of bonds and floppy motions has been an impossible task. At most, one seeks the best $Q$ among a limited class of choices of $Q$ [as in S. J. Klippenstein, Chem. Phys. Lett. 214, 418 (1993)].

${ }^{18}$ R. M. Bowman, K. B. Eisenthal, and D. P. Millar, J. Chem. Phys. 89, 762 (1988); R. M. Bowman and K. B. Eisenthal, Chem. Phys. Lett. 155, 99 (1989); D. M. Zeglinski and D. H. Waldeck, J. Phys. Chem. 92, 692 (1988); D. H. Waldeck, Chem. Rev. 91, 415 (1991); M. Lee, A. J. Bain, P. J. McCarthy, C. H. Han, J. N. Haseltine, A. B. Smith III, and R. M. Hochstrasser, J. Chem. Phys. 85, 4341 (1986); S. H. Courtney, S. K. Kim, S. Cononica, and G. R. Fleming, ibid. 78, 249 (1983); Y.-P. Sun, J. Saltiel, N. S. Park, E. A. Hoburg, and D. H. Waldeck, J. Phys. Chem. 95, 10336 (1991); J. Saltiel and Y.-P. Sun, ibid. 93, 6246, 8310 (1989).

${ }^{19}$ M. Cho, S. J. Rosenthal, N. F. Scherer, L. D. Ziegler, and G. R. Fleming, J. Chem. Phys. 96, 5033 (1992); G. van der Zwan and J. T. Hynes, J. Phys. Chem. 89, 4181 (1985); R. Jiminez, G. R. Fleming, P. V. Kumar, and M. Maroncelli, Nature, 369, 471 (1994); S. J. Rosenthal, X. Xie, M. Du, and G. R. Fleming, J. Chem. Phys. 95, 4715 (1991); L. E. Fried and S. Mukamel, ibid. 93, 932 (1990).

${ }^{20}$ J. T. Hynes, J. Phys. Chem. 90, 3701 (1986); C. van der Zwan and J. T. Hynes, ibid. 89, 4181 (1985); ibid. 89, 4181 (1985); S. Roy and B. Bagchi, J. Chem. Phys. 100, 8802 (1994) 